\title{
Custom psychosurgery
}

\author{
ERIC TURNER \\ M.B., F.R.C.S.
}

\section{Queen Elizabeth Hospital, Birmingham}

\begin{abstract}
Summary
It seems fundamentally improbable that different mental symptoms and personality disorders should be relieved by one single form of operation on the brain. The present paper describes five operations which can be performed with benefit to different types of psychiatric abnormality; namely, unilateral temporal lobectomy, unilateral or bilateral temporal lobotomy, inferior quadrant pre-frontal leucotomy, paramedian frontal leucotomy and posterior cingulectomy.

The symptoms that represent an indication for these various operations and the results of the operations on the symptom complexes are described. The proposition is put forward that only operations suitable for the precise psychiatric diagnosis should be used. A new form of analysis of sphenoidal lead examinations is described which is of primary importance in conjunction with psychiatric examination in deciding which operation a particular patient would or would not benefit from. A concept of diencephalic instability is introduced in connection with this EEG analysis to supplement the usual concept of centrencephalic epilepsy. Diencephalic instability as defined here seems to respond specifically to paramedian lobotomy, provided of course the clinical condition justifies such a step.
\end{abstract}

\section{Introduction}

Apart from the folk-lore surgery of ancient trephine holes to allow the emergence of demons, deliberate attempts at psychosurgery can be traced back to early isolated attempts at surgical treatment of metal derangements by Burckhardt in the form of unilateral multiple cortical excisions in 1890; and to Puusepp's unilateral sub-cortical section of the centrum semiovale in the frontal region of the dominant hemisphere performed between 1906 and 1910 . What might be regarded as the precursor of modern psychosurgical procedures stemmed from early observations of John Fulton \& Jacobsen (1935) and of Brickner (1934). The first bilateral operations on what are loosely termed the 'prefrontal' lobes were reported by Moniz \& Lima (1935). Bilateral lobectomy proved to be unnecessarily drastic and the effect of relief of anxiety and tension could be achieved by severing the white matter in front of the motor and premotor areas of the frontal lobes, that is behind the 'prefrontal' lobe (Freeman \& Watts, 1942). The reasons for the choice of 'prefrontal' leucotomy have been recounted many a time. Patients with disease or injury of the frontal lobes showed lack of concern. Chimpanzees observed and described by Fulton \& Jacobsen had been noted to be less prone to emotional stress, frustration and catastrophic reaction when presented with problems, and it was felt that certain 'neurotic' patients could benefit from a similar effect on their mental states. The operation of Freeman \& Watts (1942) started what ultimately turned out to be a wave of enthusiasm for the so-called classical lobotomy which severed practically the whole of the white matter of the 'prefrontal' lobes as far back as could be dared. Indications for the operation were various, and the operations themselves were largely uncontrolled by radiological means, as they were applied arbitrarily? to different sizes and shapes of skull according to set measurements. Accidents from haemorrhage, infection or misplacement were not infrequent. The psychiatric indications for the operation were at first thought to be most favourably obsessional neurosis, tension and aggressive schizophrenia. Numerous cases of schizophrenia of different sorts were leucotomized with either beneficial result or with the claim that at least some of them could now be accommodated in a better and more comfortable ward under more free conditions.

With the introduction of modern tranquillizers many of the indications for operation on schizophrenia were grossly diminished and it is seldom that schizophrenics nowadays are treated surgically, though the occasional patient benefited to such an extent that the subject of psychosurgical treatment of schizophrenia cannot even yet be considered permanently closed.

At the same time as the indications for surgical treatment of schizophrenia diminished almost to disappearing point, two other changes took place in the psychosurgical field. The first was that with increasing emphasis on the limbic lobe with its autonomic connections and its relevance to emotions and 
temperament, surgical operations became increasingly directed towards elements of this limbic lobe with the idea that the cognitive functions resided principally in the other, that is the isocortical parts, and should not be interfered with if possible. It is interesting that attempts to fractionate the section in the frontal lobes began soon after the introduction of the 'standard' leucotomy. Hofstatter, Smolik \& Busch (1945) reported favourably on operations restricted to the lower quadrant. Differential effects were sought for, and Berliner et al. (1945) described relief of 'mental tension'.

These early developments foreshadowed the directions future progress was to follow. Various forms of fractionated frontal lobotomy emerged and the ones that concentrated on the limbic structures consisted in medial frontal lobotomy and lower quadrant lobotomy which could be done either from the side or from the front. Operations from the front could be carried further back to undercut the orbital surface of the frontal lobe, the gyrus rectus or Area 13. This, and other modifications, produced results which were claimed to be good in certain kinds of case and the side effects were very much less, especially when they were well controlled by radiological means and kept forward of the dangerous areas. Although the effects were less drastic in the very modified forms, patients were more liable to relapse or recurrence of the symptoms. Nevertheless, this trend was an obvious and necessary one, and was followed by assessment of the particular symptoms that were relieved by particular modified operations.

The second change that took place was a shift in emphasis of indication for surgery. It became clear that the best results were obtained in cases of involutional depression, especially in patients with a previously robust personality, effective performers, preferably with obsessional features in their premorbid history. This group of patients was quite distinct from the group of obsessional neurotics who broke down early in life, where the obsessional neurosis was the illness itself, possibly originally a kind of defence. Obsessional features themselves proved much less tractable by the modified operations than had previously been thought. One of the reasons why they had previously been thought to be good risk cases was that they proved very resistant to various forms of frontal lobotomy, and with the fractionated lobotomy the likelihood was of incomplete relief of symptoms rather than unwanted side effects. They did rather better with medial lobotomy than lower quadrant lobotomy, but the reverse was the case with depression, which responded well to lower quadrant lobotomy but was relatively untouched by medial lobotomy.

Technical methods improved and various special attempts were made to increase the correlation be- tween symptoms and anatomical destruction. Indwelling electrodes were inserted and the effects of stimulation noted. Recordings taken to show whether the active part of the electrode was in grey or white matter, and finally small progressive lesions were made to keep to a minimum the destruction and at the same time note fractionally the effect on the individual (Crow, Cooper \& Phillips, 1961). Such methods led to increasing confidence in the effects of certain placements of the destructive lesions and, for example, in the view that lower quadrant operations were more effective for depression.

Great interest was taken in the distinction between the more primitive cortex and sub-cortical structures of the limbic lobe and the more recently developed isocortical areas. The limbic or marginal structures bordered the diencephalon and consisted of posterior orbital frontal regions, anterior and posterior cingulate gyri, hippocampus and amygdaloid nucleus, together with the overlying cortex of the hippocampal gyrus and the pyriform area. These areas were shown to have strong autonomic functions and Papez suggested that they, together with the subcortical connections of the fornix, mammillary body, mammillo-thalamic tract and anterior nucleus of thalamus with its connections with the cingulate gyri, formed the anatomical substratum of emotional experience. This clear-cut and simple theory has proved to be only partially true in man, as the hippocampus, fornix, mammillary bodies and mammillothalamic tracts are apparently concerned with recent recall of memory patterns and the laying down of recent experiences into long term memory. The other limbic structures, however, do seem to have a connection with emotional experience and most successful psychosurgical operations have been directed at these structures. Bilateral destruction of the amygdaloid nucleus or connections passing through it has been used for outbursts of uncontrollable rage and aggression, while many operations recently have involved destruction of the grey matter of the anterior cingulate gyrus, or parts of the cingulate bundles lying underneath it. My own experience has been centred on bilateral ablation of the posterior cingulate gyrus which will be described later. Various lesions in nuclei of the thalamus or even of the hypothalamus have been applied by stereotaxic means as alternatives to destruction of cortical areas with which they are known or thought to be connected, but owing to the difficulty of analysing such complicated sub-cortical structures and pathways, these methods are not in general use. Clearly, understanding of the effects of such treatments demands constant review of the information to date, correlation of the psychiatric terms of reference, accurate description of the anatomical lesions performed and comparison of the effects. Not only are 
single experiences and reports to be studied, but wherever possible, the effects of different types of operations observed by the same group of workers enable a stricter comparison to be made than is often possible when trying to compare different reports from different centres. For this reason, the present paper will now be confined to actual experiences with four different types of psychosurgical operation, the criteria that are employed to determine which operation shall be used, the comparative results on symptoms, the type of psychiatric assessment and rehabilitative programme and the type of case that has not benefited from these procedures; finally, the conclusion regarding the present psychosurgical scene will be discussed.

It must be remembered that the theories on which various psychosurgical procedures are based, such as the observations of lobectomized animals, the Papez theory of emotion, the 'visceral' brain of MacLean and the concept of the limbic lobe represent no more than working hypotheses for investigation into the possibility of helping human patients. As always in clinical practice, such efforts must be made within a strictly ethical framework and must be severely monitored at every stage to ensure that what might be a reasonable and intelligent conjecture in fact works out in practice. There are no categorical imperatives; a theory either works or it does not work. As long as it works it may be true; as soon as it ceases to work it is false.

\section{Reports}

\section{Temporal lobotomy}

In the course of investigation and treatment of temporal lobe epilepsy by unilateral lobectomy many cases emerged who had bilateral disease. Such cases were very liable to show severe personality disorder in the form of outbreaks of uncontrollable rage and as analysis proceeded, it was realized that these rages were usually of a particular kind, ushered in by experiences of sudden fear, panic or stark terror. They also suffered usually from major psychomotor epilepsy, often with an epigastric aura, from minor psychomotor attacks in the form of brief automatism, and frequently in addition progression to grand-mal attacks or generalized convulsions without remembered warning. Bilateral lobectomy has been shown by Scoville \& Milner (1957) to be dangerous, as it involves a risk of intellectual mutilation in the form of abolition of recall of recent memories and abolition of the imprinting of fresh memories into long term storage. It has been suggested that the hippocampus is involved in this process though this concept is not universally accepted. It has proved possible, however, to treat such cases by operation at or just behind the uncus of the hippocampus and in or just behind the amygdaloid nucleus through the roof of the ventricle. My own practice is to perform a type of lobotomy which usually has to be bilateral. Electrolytic lesions are placed with the aid of the Hughes stereotaxic frame after myodil ventriculography in the roof of the ventricle $1 \mathrm{~cm}$ behind the tip and in the floor of the ventricle at the same level (Figs. 1 and 2). It is not possible to separate the purely epileptic indications from purely psychiatric indications in this group as the two merge together with a considerable overlap. It may be said, however, that while no case of bilateral temporal lobe epilepsy is without its personality difficulties a number of cases of personality disorder have been treated in this way who have not shown actual epileptic attacks. In all, eighty-nine cases of temporal lobotomy have been performed for these reasons. All have been investigated by EEG and sphenoidal leads as well as radiological methods of investigation. Most have shown sphenoidal lead abnormalities in the anterior basal temporal regions, but in some the abnormality has consisted of no more than an instability without phase reversal at that site, that is without evidence of focal discharges actually arising there. In such cases the indication has been mainly psychiatric in the form of phobic rages. It should be noted that other forms of aggression are not considered to implicate the temporal lobes and aggression alone is not sufficient indication to perform temporal lobe operation. With this reservation, can be said that the effect on psychiatric symptoms i

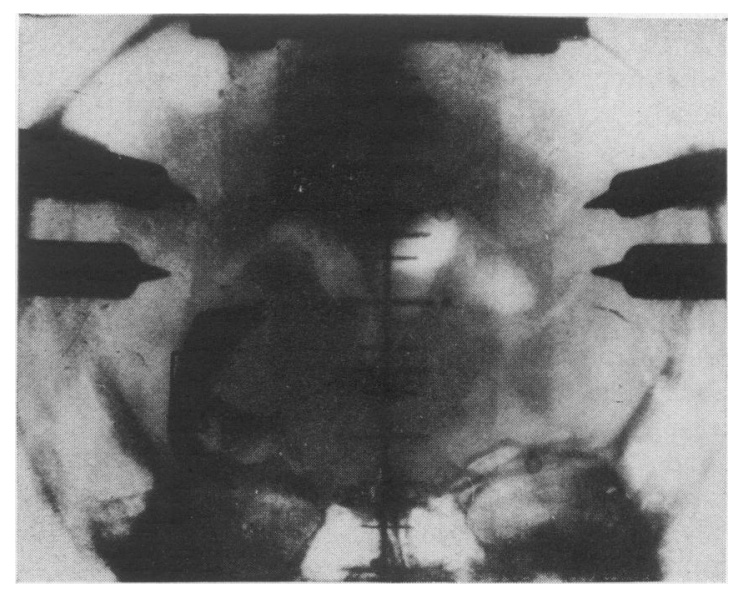

FIG. 1. Myodil ventriculogram showing the right temporal horn outlined by myodil and a line silhouette of the lesions above and below the ventricle as seen in this view. These lesions lie $1 \mathrm{~cm}$ behind the tip of the temporal horn and are tilted with the upper part of the lesion about $20^{\circ}$ forwards and the lower part about $20^{\circ}$ back of the coronal plane. 


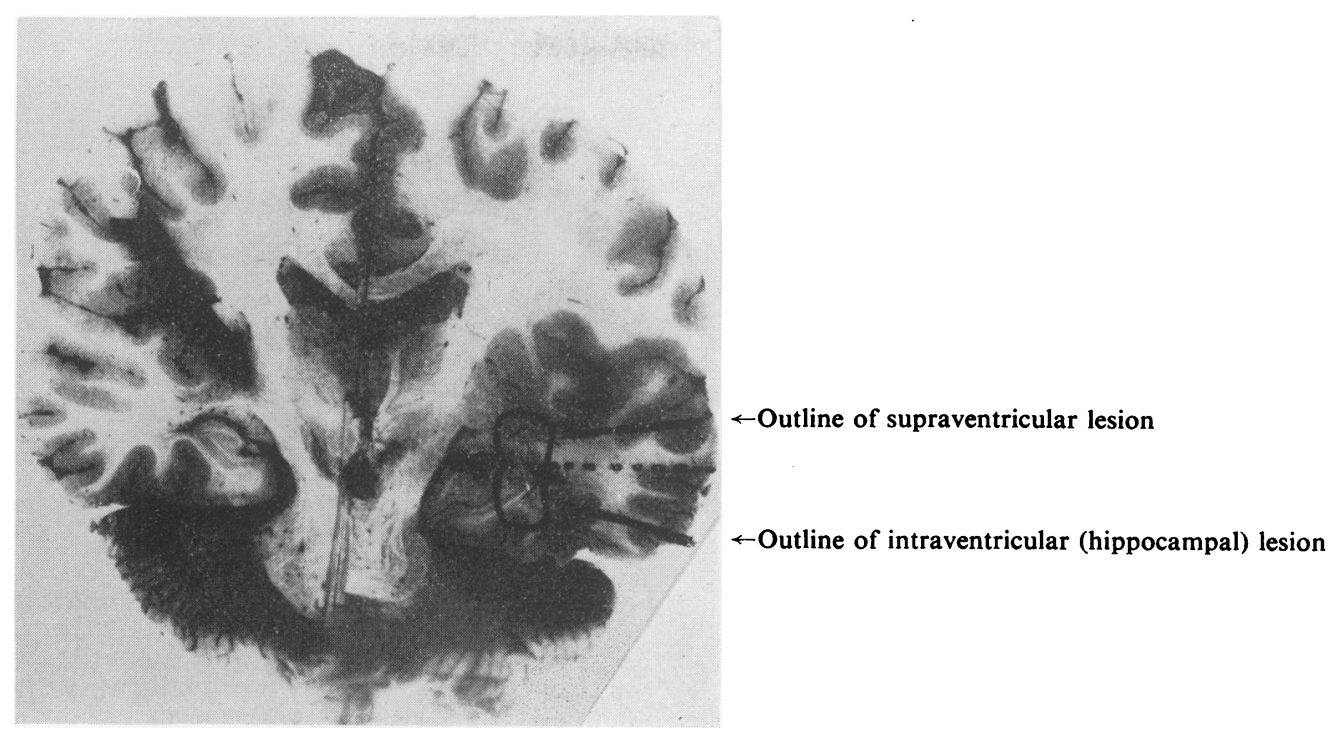

FIG. 2. Coronal section of the brain showing a line outline of the lesion in relation to the posterior edge of the uncus and just behind the amygdaloid nucleus. The lesion is $4 \mathrm{~mm}$ thick in the antero-posterior direction.

uniformly good; personality disorder of the type described is invariably improved or completely eliminated. The effects on the epilepsy is that major psychomotor attacks are usually abolished, minor psychomotor attacks diminished and generalized convulsions also reduced in frequency, duration and severity. Symptoms and personality disorders that are not improved, or are even worse, are depression and chronic truculent aggressiveness of the 'paranoid' type. By the latter is meant not paranoia with delusions, to be discussed later, but the chip-on-theshoulder resentment and hatred of the world in general that many people show and which in some patients prevents them from living in society or finding employment. Depression is apt to occur, particularly in the early months after temporal lobotomy. At first, large mood swings from elation to depression are seen but gradually they level out. During this time antidepressive drugs are useful and if depression persists after 6 months ECT may be necessary. If that is insufficient a basal frontal lobotomy has occasionally been required. Tension is reduced to some extent by temporal lobotomy but not to any great degree. Fear is abolished if it is ictal, but if it is of the frontal lobe apprehensive type as seen in hypochondria or fear for the future or of imagined possibilities it is not abolished and may not even be relieved.

\section{Posterior cingulectomy}

Following on the early frontal lobe operation the search for fractionated psychosurgical intervention caused critical examination of all parts of the limbic lobe. Fulton, whose work had started off the original operation of Moniz, was asked at a meeting where the psychosurgeon should look for an opportunity to produce an effect on temperament without disturbing intellect, and he replied that he thought the cingulate gyri represented the best chance. This was somewhat over 20 years ago and in the subsequent period of time many operations have been performed, particularly in the anterior cingulate gyri and in various levels of the cingulum, the underlying bundle of white fibres which represents a composite bundle of connections between the frontal lobe, the hippocampus, the parietal lobe, the thalamus and caudate nucleus. My own experience has been with ablation of the posterior cingulate gyrus. It is extremely difficult to compare one operation with another and clearly sometimes what is called anterior cingulectomy, that is a removal of the grey matter, involves also damage to the underlying white matter, sometimes so deeply that it amounts to a kind of medial frontal lobotomy involving the forceps minor. The result is that the literature is extremely difficult to evaluate. Anterior cingulectomy or 'cingulotomy', which is a loose term applied usually to a destructive lesion in the cingulate bundle, has been used in various conditions including obsessional neurosis and schizophrenia. The exact kind of obsessional state and the exact kind of schizophrenia are often left vague and the anatomical situation of the lesions, though on the whole better described, is not uniformly clear. I have not had success in true obses- 
sional neurosis with anterior cingulectomy, although admittedly I have not done enough cases to be certain. I failed to find in the literature a clear indication of what indications there should be for this operation, nor a clear indication of the specific effects on the individual's mentality or performance which one could expect after the performance of it. One thing seems clear, that there is no permanent effect on memory and that claims have been made of improvement in various features which can be produced by medial frontal operation. It must rest with others to support their case with a greater experience than mine.

Posterior cingulectomy, however, is a field in which I have had some experience. The indications for this operation again emerged in the course of investigation and treatment of epilepsy but epileptic criteria were quickly supplanted by psychiatric ones and most of the cases are purely psychiatric. Those indications are chronic resentful aggressiveness which prevents the individual from living in society. Such a type of aggressiveness is seen in true paranoia but is also seen in other forms of personality disorder. Clearly, the line between normal human aggressiveness and this pathological degree of it cannot be too clearly drawn, but only the most extreme examples would be even remotely considered for operation. If the cases are really well chosen the results are very good and, as in other forms of ablation rather than lobotomy, are permanent without tendency to reduction of the effect. The chronic aggressive state must be distinguished, however, from states of impulsive aggressiveness with tension, and as in other forms of psychosurgery success depends more on the assessment of the case than performance of a particular operation in all cases. In the performance of the operation a small posterior parietal bone flap is turned down on one side and the medial surfaces of the two cerebral hemispheres are exposed on the same side by simple retraction and on the other side by an incision in the falx. The posterior cingulate gyri are recorded by surface electrodes, stimulated, and then ablated by subpial suction (Fig. 3). Thirtyseven operations have been performed. The results have shown a reduction in the chronic type of aggressive behaviour which has been outlined. The side effects of the operation have included paraesthesia in one or both lower limbs which patients may find aggravating, especially if they are of the hypochondriacal tendency. There is no effect on memory and no effect on control of micturition. The symptoms which are left untouched or which are worse after this operation are panic rages, hypochondriasis and tension. I do not think depression would be improved but have not had a case with straightforward depression in this group; the nearest approach to it being hypochondriasis.

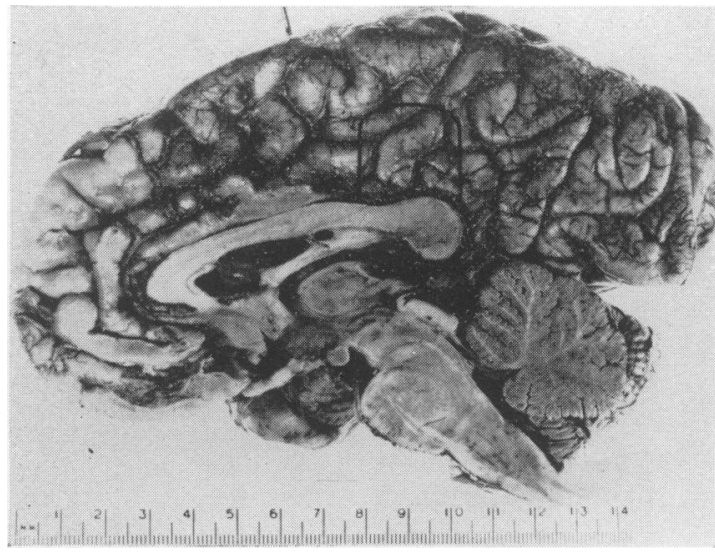

FIG. 3. Medial surface of the hemisphere as seen in midline sagittal section with the area ablated in posterior cingulectomy outlined in black. Note that the gyri turn vertically upwards here and the removal does not conform with any single gyral configuration.

\section{Lower quadrant frontal lobotomy}

This operation is done through lateral burr holes and is now performed with the aid of a pneumoencephalogram to ensure that it lies just in front of the anterior horn of the ventricle and just in front of the lesser wing of sphenoid. These two land-marks determine the plane of section, the burr hole being placed in the optimum site in relation to markersplaced on the scalp at the time of the pneumoencephalogram. The plane of section is the same as in the paramedian lobotomy to be described below (Fig. 4). It passes just in front of the genu or the corpus callosum (Figs. 5, 6 and 7), and 174 lower quadrant operations have been performed. The indications for the operation have been entirely psychiatric, though on some occasions sphenoidal lead examinations have been carried out, especially in cases where phobias are complained of.

The prime indication for this operation has been depression, and where this presents in a pure form, as in many cases of involutional depression, this has been the only indication. In young people, there has been an increasing tendency to differentiate the tension states from straightforward depression and in older age groups obsessional features may be present and tend to fall into two groups: first, the obsessional personality who breaks down into straightforward depression; secondly, the obsessional personality who becomes depressed but in addition has an increase in obsessional characteristics. Both of these groups are excellent candidates for frontal lobotomy, provided of course their illness is severe enough to prove intractable by all other conservative means, including all relevant drug therapy, and at 


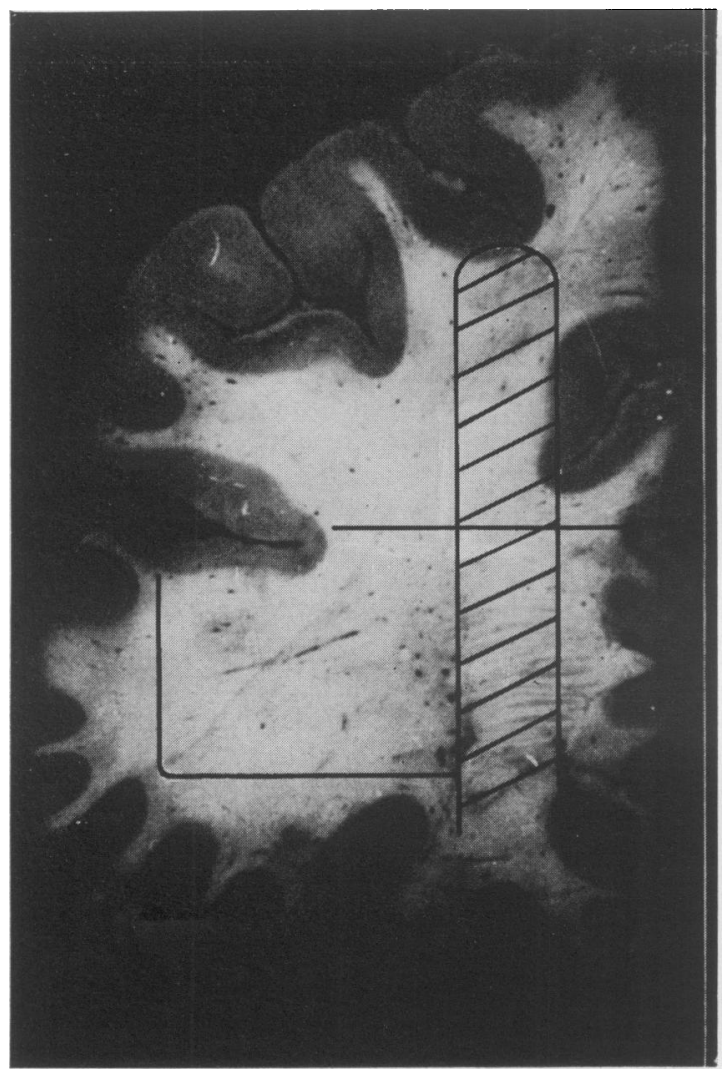

Fig. 4. Outline of the plane of section of the paramedian and lower quadrant lobotomies. The paramedian is hatched and the lower quadrant outlined in black.

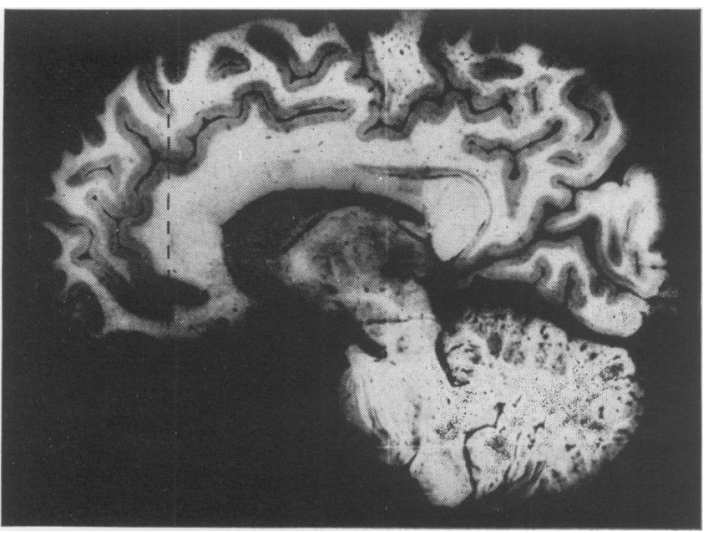

FIG. 5. Plane of section as shown in a sagittal brain section $5 \mathrm{~mm}$ from the mid-line. The pre-frontal lobotomy passes just in front of the corpus callosum. It does not of course come as far medial as this sagittal section.

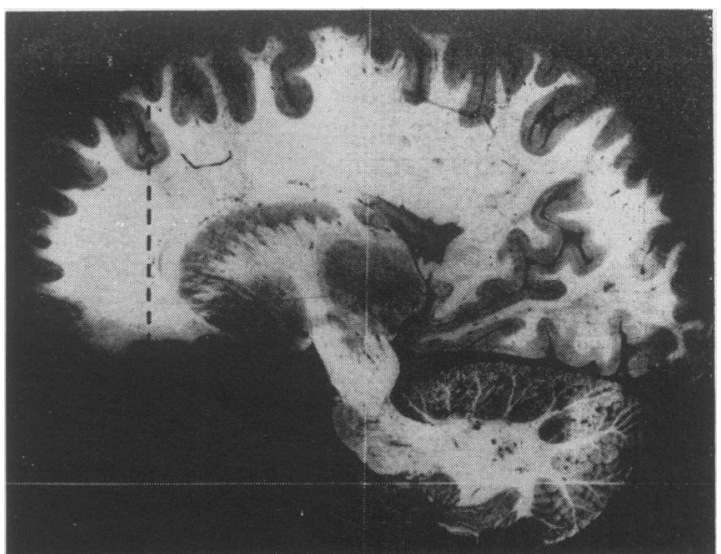

Fig. 6. Sagittal section $10 \mathrm{~mm}$ from the mid-line showing plane of section at its most medial extent.

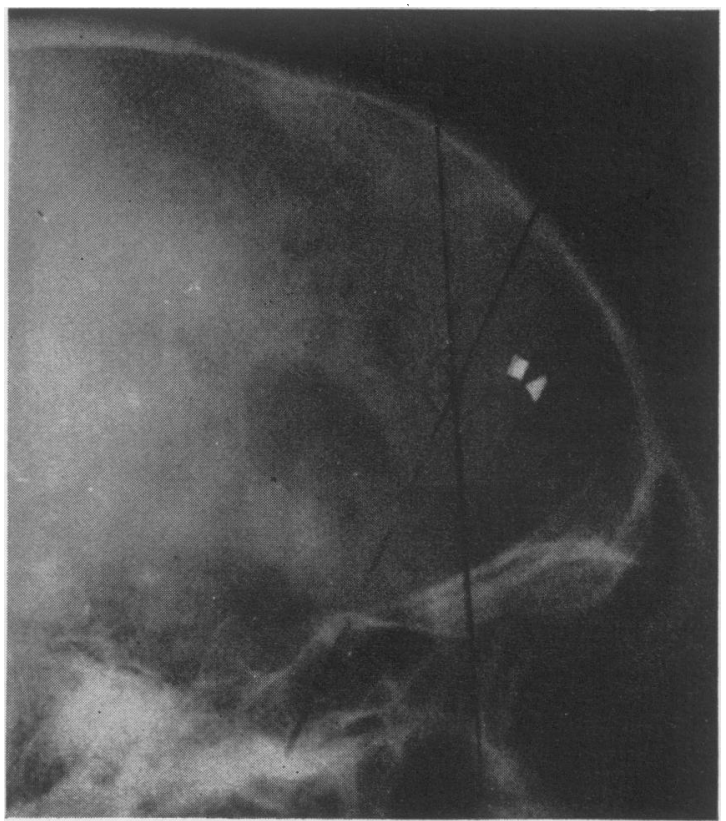

Fig. 7. Pneumoencephalogram showing the anterior horn of the ventricle and the pericallosal sulcus which form markers for the lobotomy.

least one course of ECT. Often numerous courses of ECT have been given but it is undesirable that this form of therapy should be persisted with beyond a reasonable limit.

The results of lower quadrant operations are the almost universal abolition of depression in the immediate post-operative period. If the lobotomy has been too limited, relapse has occurred, and after 
performing a very conservative lower quadrant operation for some time I have come to make it somewhat more substantial by bringing the section $2 \mathrm{~cm}$ higher than the original upper limit. This is easily done as the upper limit was previously marked by the exact line of the burr holes. Since that time, the relapse rate in straightforward depression has been much lower. Prominent obsessional features made worse by the illness or obsessional neurosis itself is not cured by a lower quadrant operation. The more prominent obsessional features are, the more necessary it is to include a paramedian lobotomy with the lower quadrant, so that it can be said that a lower quadrant does not abolish but only diminishes obsessional features in themselves. Disinhibition has not been seen in this group but it must be remembered that the cases have been chosen with great care and particular regard to their previous personality. The only form of disinhibition that has been observed is one of increased sexuality, which in one male case has necessitated the prescription of oestrogen to reduce the activity which was disturbing his spouse. Tension is not reduced and chronic aggressiveness of the 'paranoid' type is not reduced. Phobic rages are not reduced. Irritability is reduced, but of other forms of aggression the only thing that can be said is that attacks on others are less likely to be followed through. The kind of marital disharmony that stems from depression is considerably helped, however, and apart from a certain outspokenness the marriage partners usually find the patient incomparably easier to live with. Suicide is rare after such an operation though if the operation is too limited it is not entirely unknown. As can be seen by the number of cases performed this operation is one of the best known and most successful forms of psychosurgery in terms of relief of suffering. It has now stood the test of time as in various forms it has now been performed over a span of some 20 years and instead of becoming less frequent or smaller it is maintaining its frequency and maintaining its increased dimensions in my practice.

\section{Paramedian frontal lobotomy}

This operation is also of considerable standing though, in the form that I practise it now, it is comparatively recent in my own hands. Since, however, it is an operation that has been well tested by others and is now being applied according to certain well-defined objective tests, it will be described in some detail. Twenty-one such operations were performed by me in 1972.

Like the lower quadrant, the operation is performed through lateral burr holes as this approach reduces the frequency of post-operative epilepsy, which is extremely low in both of these frontal operations performed by this route. Like the lower quadrant operation its medial limit is $1 \mathrm{~cm}$ from the mid-line and in the case of the paramedian lobotomy its lateral extent is $1.25 \mathrm{~cm}$ in the plane passing in front of the lesser wing of sphenoid and just in front of the genu of the corpus callosum, which can be identified in the pneumoencephalogram by the pericallosal sulcus. The section is carried down to the base and up to the vertex just short of the piaarachnoid (Figs. 4, 5, 6 and 7). The indications for the operation are first, with this operation done in isolation, tension and impulsive behaviour usually of an aggressive nature which reflects back on the individual and renders his freedom either curtailed or in jeopardy. In other words he is not able to live in society because of his lack of control and he and others may be genuinely afraid that he may commit serious assaults or even murder. Insight is well retained when the syndrome is present in its pure form and the individual readily seeks help. The condition has to be distinguished sharply from the attitude of the habitual criminal and this is one reason why no inducement should be offered to such an individual to have surgical treatment rather than punishment for a crime. The correct type of individual will himself seek medical help irrespective of punishment for anything he has done. Indeed, he does not usually try to avoid punishment in his state of contrition. The operation is frequently employed, however, as an addition to lower quadrant lobotomy as the paramedian lobotomy has a greater effect in reducing obsessional symptoms. In obsessional people, the combined operation is not too much, as in this group of cases the danger is incomplete relief of symptomatology rather than undesirable side effects. Paramedian lobotomy may be performed alone if there are no depressive features with the obsessions, but this is seldom seen in the older age group and is confined to the difficult group of young obsessional neurotics who often present with tension rather than depression. It is interesting that the assessment of this group emerged out of investigation of patients with personality disorders in general with or without epilepsy. Such patients, who show extreme tension, frequently describe attacks that may be classed by onlookers as hysterical attacks and may be ushered in by overbreathing, and in the pure form they do not present convulsive features. In the course of their investigation sphenoidal leads were performed and it was noticed that these people showed, to a somewhat less marked degree than in centrencephalic epilepsy, a phenomenon under pentothal anaesthesia which has been described as diencephalic instability, to distinguish it from but at the same time show similarity to centrencephalic epilepsy. This feature is shown by the presence of high voltage sharp and slow waves which are normal at isocortical areas under moderate pentothal anaesthesia but which in 
these cases are synchronous on the two sides between the frontal bases and parietal convexities (Fig. 8). When this type of EEG record is shown in sphenoidal leads and is accompanied by appropriate psychiatric indications, the outcome of the operation can be anticipated with a considerable degree of confidence. Tension is reduced together with impulsive aggressiveness. Depression is only slightly reduced and paranoid or chronic grudges may be preserved, though they are not acted out in terms of impulsive aggression. The operation has not been performed with clear-cut temporal lobe foci but it would not be expected that the temperamental disorders associated with such temporal lobe epilepsy or the psychomotor attacks themselves would be reduced.

Since these operations are not mutually exclusive, combinations of them may on occasion be indicated. In such a case an interval of at least 6 months is normally left before a complete re-assessment of the case shows the evidence necessary to suggest an extension of operation. Thus, depression remaining after temporal lobotomy will respond satisfactorily to lower quadrant frontal lobotomy. In such a case every fresh attempt is made to control the depression by other means before further surgical intervention is contemplated. Mood swings after temporal lobotomy are relatively severe at first but tend to settle down, so that the first requisite is the passage of a reasonable amount of time. If depression is still a disabling feature in spite of pharmacological antidepressants, ECT may be given a trial, though this is not usually continued or repeated unreasonably. After temporal lobotomy also, chronic paranoid aggressiveness may continue to prevent a patient from living and working freely in society and in extreme cases a posterior cingulectomy may be considered. When posterior cingulectomy has been performed as a primary operation, tension and impulsive aggressiveness may persist and the paramedian lobotomy may have to be considered. Combinations of lower quadrant and paramedian frontal operations have already been discussed.

Conditions which prove intractable or are relatively resistant to any of these operations, or combinations of them, include obsessional neurosis in young people, simple schizophrenia and hysterical personalities with life-long inadequacy, work-shyness and attention-seeking symptoms. For obsessional neurosis of the defensive type, anterior cingulectomy or 'cingulotomy' has been advocated and reported favourably by other workers but I have not had sufficient experience to come to a final conclusion myself. In my hands, anterior cingulectomy, in the sense of removal of grey matter only, has not been successful, and section of the cingulum bundle at various levels in severe cases of this type of

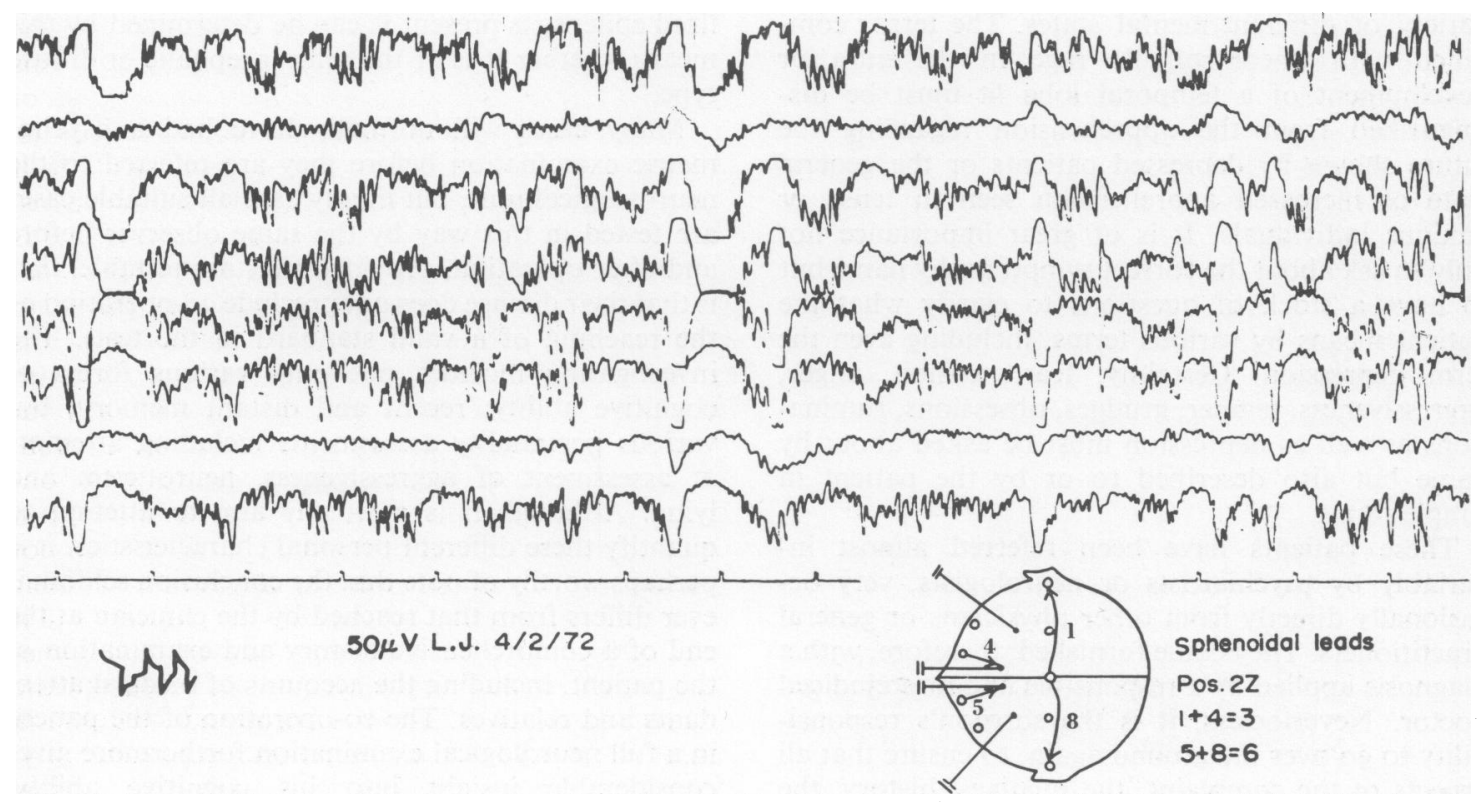

FIG. 8. Sphenoidal lead EEG performed under pentothal anaesthesia to show the synchronous high voltage sharp and slow waves in channels $1,3,4,5,6$ and 8 . These represent the frontal bases and the parietal convexities. In this record a form of analysis is used in channels 3 and 6 which represent the addition of channels 1 and 4 and 5 and 8 respectively. True electrical synchrony is thus demonstrated and the manner of the write-out ensures that the feature can be readily detected and actually counted without trouble. 
illness, has not come within my experience adequately for me to speak about it. I would only make the plea that the diagnosis should be defined or described in terms that could be clearly recognizable to other workers and that any anatomical operation should be equally clearly described. Only when this is done can various conflicting reports be reconciled and the meaning of the results be appreciated. Whether simple schizophrenia will ever be a surgical problem is open to doubt. Its very nature and pathology, if there is any organic pathology, have not been defined. Empirical attempts at treatment have been either unsuccessful or inconstant, so we must for the moment accept that treatment must await diagnostic understanding. Paranoid schizophrenia and various states resembling schizophrenia in many respects, but with a retention of affective keenness and suffering, may be a different problem altogether and the question of surgical treatment should be considered on its merits in individual cases. Such states may be seen as secondary symptoms in temporal lobe epilepsy but a true schizophrenia seems never to be helped by temporal lobe surgery. Some benefit may be seen from posterior cingulate or paramedian operations in states of mind which have a certain kinship with paranoid schizophrenia and true paranoid schizophrenia may be benefited to some extent by such operations.

Phobias, like aggression, may be a term covering a variety of different mental states. The terror combined with uncontrollable rage in the aura or development of a temporal lobe fit must be distinguished from the apprehension regarding the future shown by depressed patients or the general state of increased apprehension seen in tense or anxious individuals. It is of great importance not only to ask about the correct symptoms by name but to have a stock of questions to clarify what the patient means by various terms, including even the term depression. Certainly, fear, tension, anger, aggressiveness, temper, grudges, obsessions, ruminations as well as depression must be asked about by name but also described to or by the patient in simple terms.

These patients have been referred almost invariably by psychiatrists or neurologists, very occasionally directly from other physicians or general practitioners. They come furnished, therefore, with a diagnosis applied by a responsible and unprejudiced doctor. Nevertheless, it is the surgeon's responsibility to go over the ground again, to ensure that all aspects of the complaint, the relatives' history, the previous personality and the gravity of the present state are adequately understood. In all the forms of psychosurgery the most promising case has a decipherable personality apart from his illness. A previously adequate or superior performance is a great boon and a warm and attractive personality outside the area of psychiatric symptomatology a great advantage, for if certain symptoms are reduced or abolished some mainspring of activity in the form of another type of emotional drive is fundamental to post-operative success. The attitude of relatives, the nature of the domestic situation, the availability of skilled psychiatric rehabilitation are all important points in deciding whether an operation is advisable or not. Once a case is referred, previous medical notes are examined, and the patient is interviewed together with someone who knows him in a hospital environment and a relative who has known him in the past and will be concerned with his future. Routine physical examination including straight $\mathrm{X}$ ray of the skull and chest, straight EEG and the usual neurological examination are supplemented by sphenoidal lead examination under pentothal anaesthesia. In the first place, this is usually performed on a day patient basis. In cases of temporal lobe epilepsy, this is repeated at least once more as a rule before any form of surgery is undertaken. In the other cases it has not been found that the condition of diencephalic instability is at all likely to be different on other occasions, and this examination is not therefore always repeated in those other cases. By means of the sphenoidal lead examination, unilateral or bilateral temporal foci can be picked-up, the featureo of diencephalic instability is recognized and if generalized epilepsy is present it can be determined by this means whether it is of the centrencephalic or diffuse type.

Many cases will normally have had a psychometric examination before they are referred to the neurosurgical unit, but in any case all suitable cases are tested in this way by the same observer before and after operation, provided they are testable, that is that their disease does not preclude co-operation or the reaching of a valid standard in the tests. The investigation includes not only various forms of cognitive ability, recent and distant memory, but various personality assessments including attempts at assessment of aggressiveness, neuroticism and lying. Although it is a worthy aim to attempt to quantify these different personal characteristics, it is perhaps worthy of note that the conclusion seldom if ever differs from that reached by the clinician at the end of a comprehensive history and examination of the patient, including the accounts of medical attendants and relatives. The co-operation of the patient in a full neurological examination furthermore gives considerable insight into his cognitive ability, especially if a few simple mental tests are included. The examinations after operation are performed in the first place within a few days of the operation and then after many months' delay. Opportunity is taken to re-examine the patient after longer intervals 
when the chance presents itself. In this way, it has been possible to determine that none of the operations described causes any significant cognitive depreciation. Unilateral temporal lobotomy can cause trivial and temporary effect on new random learning in the dominant and non-dominant hemispheres. Bilateral lobotomy does not cause the severe effect on recent memory that bilateral lobectomy has done, and since the lobotomy compares to some degree with amygdaloid stereotaxic lesions this freedom from serious effects even from bilateral lesions has been confirmed by many workers. The frontal operations produce no detectable effect on the tests, though performance may be improved by reduction of the disabling psychiatric symptoms. In particular, there has been no permanent effect on memory from the paramedian operation. The temporary effects of this operation are, however, more profound than those seen after the lower quadrant operation. The temporary effects last up to 3 weeks, usually 2 weeks, and include euphoria, nocturnal confusion, incontinence and sometimes diurnal confusion and even hallucinations. Relatives are warned of the possibility of these disturbing and alarming symptoms and are re-assured that they are of temporary nature and are normally followed by a good result. These more bizarre post-operative symptoms are seen typically in older women who have presented with agitation rather than pure depression and who have not had obsessional features in their pre-morbid personality. Screaming agitation is the syndrome most liable to be followed by these florid postoperative symptoms. Nevertheless, they are of a temporary nature, quickly settle down and then allow the patient to be rehabilitated in the usual way. Posterior cingulectomy is not followed by any kind of memory defect, contrary to what might have been a theoretical anticipation. Rehabilitation presents no problem and psychometric testing reveals no intellectual change, other than an increase in co-operation in the tests.

Rehabilitation after all these operations is important but can be conducted along orthodox lines of occupational therapy and industrial rehabilitation, re-training or simple return to previous employment in the case of temporal lobotomies or posterior cingulate operation. The patients are advised not to return to work until at least 6 weeks have passed since the operation, but equally they are not encouraged to stay unemployed any longer than this if it can be avoided. Patients who have been unemployed for many years are of course difficult to place and long unemployment with a generally inadequate pre-morbid record are factors that militate against successful reemployment. The frontal operations demand a more organized type of rehabilitation. For 3 weeks this should be within a hospital environment and should be conducted on a symptomatic basis. If the patient tends to lie or sit around, attempts are made to occupy him and get him into the habit of getting up and about; if he is confused and incontinent then of course he is simply nursed through this period until rehabilitation can start. This then takes the line of occupational therapy followed by day hospital attendance, re-training if necessary and finally restoration to a job within his or her capabilities, either in a protective environment for a year or so, or in the ordinary industrial environment. It is most important that the patient should not be restored to his home unattended too early. Six weeks of close supervision are the minimum and thereafter reasonable habits of activity should be demanded, especially in the first few months. During this time the patient is trainable and fresh habits can be laid down. Frequently, patients resist the idea of a mental hospital for rehabilitation. There is no point in arguing with them before the operation is done, but afterwards it is explained to them that this is a form of convalescence, that it is most important for their well-being and normally they accept the suggestion at that time without distress, especially when it is put reasonably to them that it is a temporary stage. Relatives can help greatly in the rehabilitation and they should be encouraged, informed and instructed as to the right way to go about things by everyone who comes in contact with them-nursing staff, medical staff, surgeon and psychiatrist. There is no point in approaching this stage in the passive frame of mind of a detached scientific observer. The detachment can come in comparing the results of vigorous rehabilitation before and after surgical treatment.

Depressive patients can relapse after insufficient operations. With the present extent of operation, however, relapse has not been so frequent and, with the aid of sphenoidal lead assessment to determine the need for paramedian lobotomy or paramedian extension, it looks as though the relapse rate will be gratifyingly low, judging from the comparatively complete relief of serious aspects of the symptomatology in those cases that have been observed so far. My experience of this particular operation is too short for me to make final pronouncement on this score.

\section{Conclusion}

Looking on the broad aspect of psychosurgery, especially in the last 10 years, one may concede that the limbic concept is essentially justified. Operations based on this concept have been uniformly successful in avoiding undesirable effects on intellectual capacity. It is beginning to appear that certain effects on temperament are predictable and can be used as criteria for selecting individuals for particular operations. Some of the early theoretical concepts have not 
stood the test of time; for example Papez's 'emotional circuit' as originally published is too inaccurate for straight reproduction as any basis for surgical treatment. The division of the limbic system into two parts, one for emotion and temperament and one for memory, is justified and although one system has a tremendous effect on the other the results of ablative or destructive operations on the two parts of the system are quite different. Far from the last word having been spoken on this theme one might rather say that only the first word has been spoken; nevertheless, the broad outline of the circuit diagram is beginning to emerge. I picture it as a system of goal-seeking circuits vying for temporary command of the diencephalic diffuse projection system with awareness occurring principally on the sensory side of the arc.

In psychiatric terms, although some answers are beginning to be formulated there are many questions completely unanswered. The nature of schizophrenia is the most prominent example, but obsessional neurosis of the defensive type in the young inadequate individual is also more difficult to treat successfully than was appreciated. The nature of sexual perversions in neurological terms is a question which remains obscure. Is it an obsession, a compulsion, a displacement activity or is it something more subtle altogether? Assuming that animals including man have both forms of activity available, the neural mechanisms are not necessarily distinct in visible anatomical terms, but the individual's inability to switch over to the normal pattern of behaviour may really be some completely different psychiatric syndrome, more like an obsessive compulsive act.

Aggression, tension and fear are too complex emotional experiences to be equated, without further definition, from one patient to another. Some forms of each are amenable to one form of psychosurgery, others to other forms. There will be many other reviews in the future reporting further progress in the search for operations that are ideally suited to an individual patient's symptoms; only by pooling the experience of many people can a full picture be built up of the possibilities of psychosurgery. Unfortunately, many reports confine themselves merely to glowing accounts of the panacea represented by one operation. It is unlikely, however, that the future of psychosurgery lies in any one promised land and psychosurgeons ought to be trained to perform some or all of the different generic types of operation and above all should be able with psychiatric help to choose the correct patient for the correct operation. This will demand agreement, either general or localized, as to what these indications are, but even before that stage is reached it demands a common language between psychiatrists and surgeons, capable of being expressed in simple English that can be understood by patients, relatives and doctors alike. While it is desirable that the medical language should be precise enough to be incapable of misinterpretation, medical jargon is far from meeting that requirement and is of course completely incomprehensible to successive waves of naïve patients and their families.

It is to be hoped that similar reports in 10 years' time will show a considerable progress in understanding and sophistication. They might even show some advance in understanding the mental illnesses that at present defeat us.

\section{Acknowledgments}

Thanks are due to numerous colleagues who have helped in dealing with these difficult problems; in particular to my psychiatric friends who have helped, guided and educated me to whatever understanding I have of the criteria necessary for these particular surgical exercises. In particular, I should like to thank Dr Derek Anton-Stephens, who must, however, not be held responsible for any psychiatric gaffes I may have committed. I am grateful also to all the members of the Neurosurgical Unit, whose forbearance has been sorely tried on many occasions; and to the technical staff who have helped me, particularly Miss Audrey Phillips who initiated me into the mysteries of practical EEG interpretation and Mr Michael Grainger who has been indispensable through the years in evolving and carrying out the various novel procedures. Without all of them, none of the work would have been possible.

\section{References}

Berliner, F., Beveridge, R.L., MAyer-Gross, W. \& MOORE, J.N.P. (1945) Prefrontal leucotomy. Report on 100 cases. The Lancet, ii, 325.

BRICKNER, R.M. (1934) The role of the frontal lobes in intellectual function. A study based on a case of partial bilateral frontal lobectomy. Archives of Neurology and Psychiatry, 31, 1118.

BurCKHARDT, G. (1890) Ueber Rindenexcisionen als Bietrag zur operativen Therapie der Psychosen. Allgemeine Zeitschrift für Psychiatrie und ihre Grenzgebiete mit Beilage Zeitschrift für psychische Hygiene, 47, 463.

Crow, H.J., CoOPER, R. \& PHillips, D.G. (1961) Controlled multifocal frontal leucotomy for psychiatric illness. Journal of Neurology, Neurosurgery and Psychiatry, 24, 353.

Freeman, W. \& Watts, J. (1942) Psychosurgery. Charles C. Thomas, Springfield, Illinois.

Fulton, J.F. \& JACOBSEN, C.F. (1935) Functions of the frontal lobes; a comparative study in monkeys, chimpanzees and man. Advances in Modern Biology, 4, 113.

Hofstatter, L., Smolik, E.A. \& Busch, A.K. (1945) Prefrontal lobotomy in treatment of chronic psychoses; with special reference to section of the orbital areas only. Archives of Neurology and Psychiatry, 53, 125.

Moniz, E. \& Lima, A. (1936) Premiers essais de psychochirurgia; technique et résultats. Lisboa Médica, 13, 152.

PAPEZ, J.W. (1937) A proposed mechanism of emotion. Archives of Neurology and Psychiatry, 38, 725.

PuUSEPP, L. (1937) Alcune considerazioni sugli interventi chirurgici nelle malattie mentali. Giornale della reale Accademia di medicina di Torino, 100, 3.

Scoville, W.B. \& Milner, B. (1957) Loss of recent memory after bilateral hippocampal lesions. Journal of Neurology, Neurosurgery \& Psychiatry, 20, 11. 